\title{
Effects of Contextual Integration on Recall of Pictures by Older Adults
}

By: Denise C. Park, Anderson D. Smith, Roger W. Morrell, J. Thomas Puglisi, and William N. Dudley

Park DC, Smith AD, Morrell RW, Puglisi JT, Dudley WN. (1990). Effects of contextual integration on recall of pictures by older adults. J Gerontol, 45(2), P52-7.

Made available courtesy of OXFORD UNIVERSITY PRESS:

This is a pre-copy-editing, author-produced PDF of an article accepted for publication in The Journal of Gerontology following peer review. The definitive publisher-authenticated version is available online at http://geronj.oxfordjournals.org/

\section{***Note: Figures may be missing from this format of the document}

\begin{abstract}
:
This study investigated age-related differences in the ability to utilize integrative relationships between target and context as a memory support by directly manipulating the relationship between a target picture and context. We hypothesized that as the active integration required between target and cue increased, age differences would increase. Old and young adults were instructed to remember target pictures, each presented with a pictorial cue. The cue/ target relationship was one of three types: categorically related (high integration condition), visually interacting (high integration condition), or unrelated and noninteracting (low integration condition). Cued recall of the targets was tested. The results indicated that the poorly integrated target-context relationship produced the largest age difference, supporting the integration hypothesis.
\end{abstract}

\section{Article:}

It has been proposed that old and young adults differ in I their use of contextual information in the memory environment. For example, Craik and Simon (1980) have suggested that memory performance of older adults is worse than that of young adults because they encode at a general level and have difficulty in effectively using specific contextual information (i.e., the general encoding hypothesis). Park, Puglisi, Smith, and Dudley (1987) and Puglisi, Park, Smith, and Dudley (1988) have shown, however, that elderly adults do use specific contextual information effectively in both the pictorial and verbal modalities under a number of different presentation conditions, including a divided attention task. Thus, the general encoding hypothesis appears to explain the relationship among context, memory, and age for only a narrow range of experimental conditions.

Context has been operationally defined in many different ways in memory research, and precise definitions have rarely been provided. Generally, it appears that context has been viewed as either experimentally presented stimulus cues or as extra-stimulus environmental information present at encoding, at retrieval, or at both times. This contextual information is conceptualized to provide some type of memory support for the target information that must be recalled or recognized. In most cases, subjects' attention has been explicitly directed toward the cues, but 
there are cases where awareness of the cues is implicit, as in studies examining the effects of room change on memory (Smith, 1979, Fernandez \& Glenberg, 1985).

Studies in the aging literature have typically used procedures involving experimentally presented stimulus cues, but have differed in how subjects were signaled to use the cues. In some cases, the experimenter has directed subjects' attention toward the contextual cues at encoding through the use of orienting tasks (Craik \& Simon, 1980). Other studies have required subjects to study the contextual cues as part of the memory task and presented them later as retrieval cues (Hess, 1984; Canestrari, 1968; Park, Puglisi, \& Smith, 1986). In these latter studies, one could conceptualize the contextual information to be part of the target at encoding because subjects are instructed to study all of the information and do not know that part of the stimulus will serve as a cue later, at retrieval. In such studies the contextual information actually serves as context only in the retrieval phase of the study, where it is presented as a cue to help subjects retrieve the remaining target information. With respect to these procedural variations, the present study involves the use of context that is part of the experimentally presented stimulus, and the subjects are aware of the distinction between target and context at all times. We have chosen not to use an extrastimulus manipulation of context (e.g., environmental room cues) because the effects produced by such manipulations tend to be weak and somewhat unreliable (Fernandez \& Glenberg, 1985). To ensure that the contextual information is separate from target information, the cue and target information are perceptually distinct in the present study and the target is clearly identified for the subject. Additionally, rather than explicitly directing subjects' attention to the cues and the relationship between the cues and target information, we use a preexperimental cued recall training session so subjects learn how the cues will be used relative to the target information. Thus, subjects learn that contextual cues present at encoding will be presented later at retrieval, but they are not formally directed as to how to use this information. This procedure permits us to evaluate how elderly adults choose to use contextual information when they are aware that its use contributes to adequate performance.

In the present study, all of the stimuli presented, both cues and targets, were pictures. We chose to use pictures as stimuli for two reasons. First, much of the context in our everyday memory environment is visual in nature, yet there is little research available on the use of nonverbal or pictorial context by older adults. Second, the present study focused on the use of integrative relationships between targets and cues, and the use of pictures permitted us to achieve the integration of targets with context in two different ways: (a) by manipulating the conceptual relationship between target and cue and (b) by varying the degree of a physically interacting relationship between target and cue. The two different manipulations of the integration variable in this fashion will help provide convergent validity for the construct of integration.

Our specific hypothesis with respect to integration was that contextual information that is wellintegrated with a target will serve as an effective memory support for older adults and that they will profit from it more than younger adults. Conversely, if contextual information is poorly integrated with a target and requires active subject-controlled integration or mediation, we expect that older adults will be disadvantaged relative to young adults. These predictions are in agreement with Craik's (1983) notion that age differences in memory tasks become magnified as the requirement for self-initiated processing increases. We have defined integration to be a meaningful relationship between target and context that is readily activated. Thus, according to 
this definition, target and context would be viewed as highly integrated and not requiring much subject-controlled integration if their meanings overlapped categorically or perceptually. There is a substantial literature suggesting that forming categorical relationships requires little selfinitiated processing for integration. For example, Howard, McAndrews, and Lasaga (1981) reported that meaning is automatically activated in both old and young, so that integration of two related items would require little effort. Similarly, two unrelated pictures could be highly integrated (have a shared meaning) if they were presented in a physically interacting relationship (e.g., a cat on a table), resulting in a unitary encoding of the two items. Both of these methods for operationalizing highly integrated target-cue relationship were used in the present study. Poorly integrated target-context relationships are represented by two unrelated items (a target and cue) that are presented side by side rather than in an interacting relationship, and thus require selfinitiated processing to form a relationship.

Several studies in the paired-associate tradition have examined the role of age and contextual integration that relate to the present study. Zaretsky and Halberstam (1968) studied the effects of similarity on paired-associate acquisition with young and old and reported that the semantic integration facilitated memory. Canestrari (1968) provided old and young adults with experimenter-supplied integrations in both the verbal and visual mode for paired associates. Subjects received unrelated stimulus-response terms and then were provided with (a) a sketch of the items in an interacting relationship; (b) integrating phrases connecting the two items, or (c) no integrations. He found that older adults were more facilitated by the integrations than young adults and that the verbal and visual integrators did not differ in effectiveness. Unfortunately, due to a number of methodological problems, it is difficult to interpret the meaning of these results with respect to age and the use of contextual information. First, Canestrari had virtually errorfree performance in young adults in the control condition, so it is not clear if the age interaction only occurred due to ceiling effects. Second, he confounded acquisition time with age by permitting self-paced acquisition and not reporting measures of stimulus duration between the two groups. It is highly probable that the older subjects took longer. Third, subjects were required to attend to both the stimulus materials and the experimenter-supplied integrating materials at the same time, and it is not clear how they allocated their attention. Finally, subjects studied both target and context as target information, so the contextual manipulation occurred only at retrieval.

The present study corrects for these methodological problems by (a) using materials where neither group is at ceiling; (b) presenting acquisition items for a fixed time period; (c) presenting stimuli where integration is varied in two ways - first, by presenting different types of targetcue relationships, and second, by presenting experimenter-supplied verbal integrations of the target-cue relationships; and (d) training subjects to use information as context at both encoding and retrieval.

In order to address these issues, the performance of young and old adults was measured in a between-groups design. Subjects in all conditions studied the same target items, but the relationship between target and cue varied in three ways between groups: (a) Control — target and cue unrelated; (b) Interacting - the unrelated target and cue were presented in a visually interacting relationship; or (c) Related - target and cue shared a semantic relationship. Additionally, half of the subjects in each condition received sentences presented aloud by the 
experimenter; these provided an integrating description of the target and context. 1t was expected that age differences would be largest in the control condition without integrating sentences.

However, if older adults can profit from experimenter-supplied integrations, one would expect that performance would improve in the control condition, particularly for the elderly, when the experimenter-supplied integrators were added.

\section{METHOD}

Subjects. - There were a total of 169 individuals in this experiment, 73 old adults (12 males and 61 females) and 96 young adults (41 males and 55 females). Young adults were college students enrolled in psychology classes and older adults were healthy, community-dwelling individuals, aged 60 or older, who were volunteers from local senior and church groups. The young adults had a mean age of 18.64 years, and the older adults had a mean age of 70.79 years. The mean vocabulary score on the Gardner-Monge (1977) vocabulary test was 20.42 for the old and 14.10 for the young, a significant difference, $\mathrm{t}(167)=8.30, \mathrm{p}<.001$. Subjects were also administered a perceived health assessment instrument (Duke University, 1975). The proportion of young adults rating their health as "better," "the same," or "worse" than others their age was .42, .53, and .06 respectively. The same figures for the older adults were, in order, .62, .31, and .07.

Stimulus materials. - All target stimuli used in the 

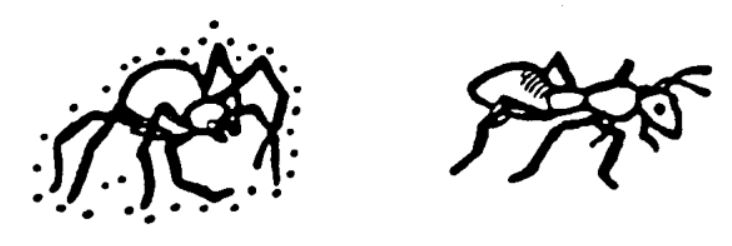

\section{Related Context and Target}
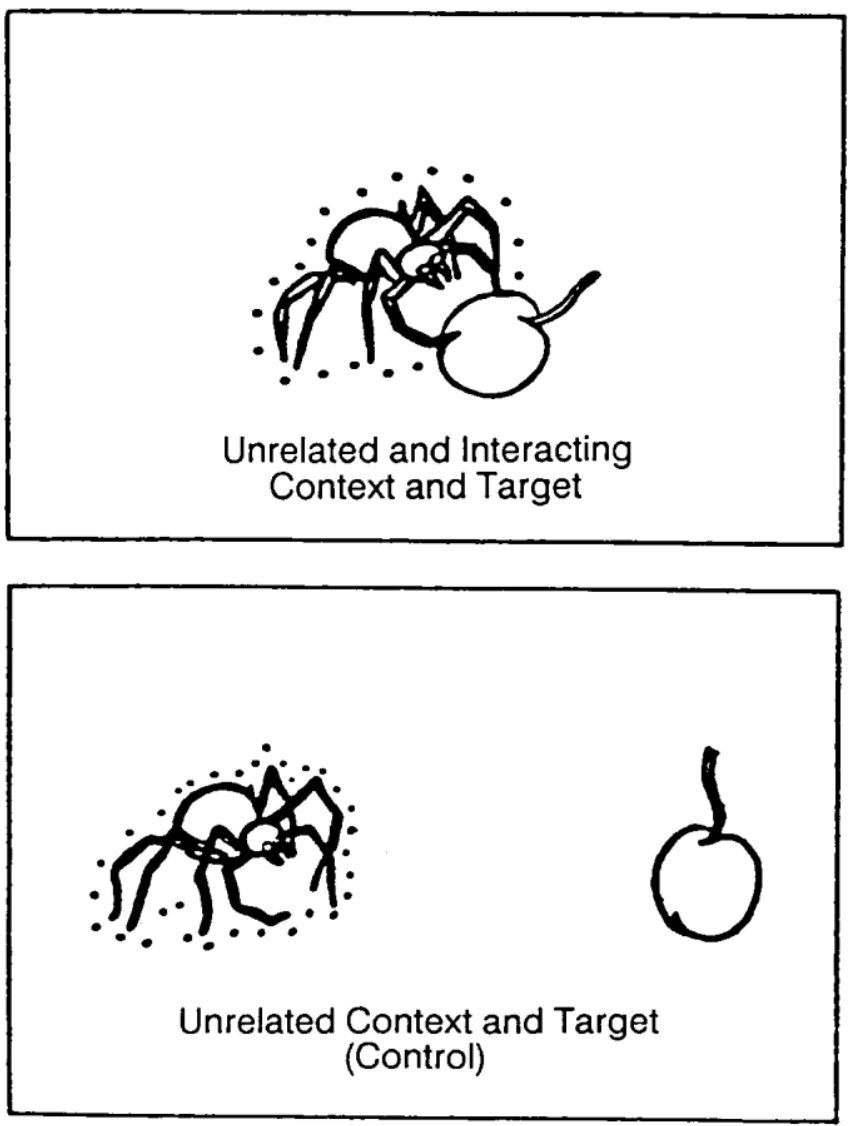

Figure 1. Sample stimuli presented for study during acquisition.

experiment were simple line drawings of concrete objects, and all were presented in the context of another picture. The target item was always drawn with heavy dark lines and then delineated with tiny dots surrounding it. Thus, subjects could readily discern which item was target and which was context, even in the interacting condition.

A total of 32 items selected from the Snodgrass and Vanderwart (1980) picture norms were selected as target items. The basis for selection as a target item was that Snodgrass and Vanderwart reported high name agreement for the item. The 32 target items were invariant across all conditions. There were three different types of context pictures associated with each 
acquisition item: unrelated (control) context, related context (same conceptual category), and unrelated-interacting context (see Figure 1 for examples). Unrelated context items were pictures that bore no obvious categorical or functional relationship to the target item (low integration condition), whereas related context cues (semantic integration) were members of the same conceptual category as the target item (e.g., "ant" and "spider" are both bugs). The context item and target were items of approximately the same size in the real world, so that an ant would not, for example, be presented with a bus or elephant as a context item due to size discrepancies. The unrelated interacting context cues were designed to be perceptually rather than semantically integrated with the target item. They were the same context items used for the unrelated (control) condition, except that they were now presented interacting with the target item. The interaction of the context with target was such that neither item's structure was changed, (e.g., a nondescript dog in the control condition would not be portrayed as jumping or barking in the interacting condition). However, partial occlusion of one item by another was permitted. As mentioned earlier, the target item was always drawn with heavy dark lines and then delineated with tiny dots surrounding it, easily distinguishing it from the context picture. The target and context picture were presented equally often on the left or right side of the slide, whereas the interacting target/context pair was always centered on the slide. Additionally, sentences that integrated and described a relationship between the target and context were created to be read aloud during stimulus presentation at acquisition. All the sentences were of the same format (noun, verb, object with adverbial or adjectival modifier). An example of an integrating sentence for the spider and ant displayed in the top panel of Figure 1 is "The spider ate the ant," whereas the integrating sentence for the lower two panels would be "The spider ate the cherry."

Design. - The study had 12 between-group conditions created by factorially crossing age (young and old) with verbal integration (presence or absence) and cuing condition (control, related, interacting).

Procedure. - Subjects were tested in small groups of two to five. Prior to the actual experimental session, subjects participated in a four-item pretraining task that matched the encoding conditions to which they were assigned. Subjects studied four cue/target pairs, performed a brief distractor task, and then had a cued recall test of the four items. Thus, subjects were aware that the contextual stimuli were important for adequate performance. During acquisition, 32 target items with context were presented at a 5-sec rate via a Carousel projector. All subjects were instructed to study the target item and were informed that relating the target to the cue might help them remember the target. Additionally, subjects in the integrating sentences conditions were also told that the experirnenter would provide them with a sentence that related the two items together, to help them remember. Subjects in the verbal integration conditions listened to sentences integrating the target with the contextual cue at the same time the item was displayed. No 
Table 1. Effects of Age and Context on Words Recalled and Words Intruded

\begin{tabular}{ccccccccc}
\hline \hline & \multicolumn{6}{c}{ Condition } \\
\cline { 2 - 3 } & \multicolumn{2}{c}{ Control } & & \multicolumn{2}{c}{ Interacting } & & \multicolumn{2}{c}{ Related } \\
\cline { 2 - 3 } \cline { 8 - 9 } \cline { 8 - 9 } & Young & Old & & Young & Old & & Young & Old \\
\hline Words recalled $^{a}$ & 16.18 & 5.79 & & 14.53 & 9.66 & & 23.78 & 16.59 \\
$S D$ & $(5.82)$ & $(4.88)$ & & $(5.93)$ & $(6.16)$ & & $(3.93)$ & $(6.42)$ \\
Words intruded & 3.72 & 5.04 & & 4.09 & 4.96 & & 3.00 & 6.91 \\
SD & $(3.94)$ & $(3.51)$ & & $(3.43)$ & $(2.93)$ & & $(2.87)$ & $(3.49)$ \\
\hline
\end{tabular}

aMaximum total of 32 words. Each mean represents 32 different subjects for young adults and 24 different subjects for old adults except for one condition, which had 25.

sentence took longer than the 5-sec presentation interval to read aloud. Following acquisition, a 3-minute distractor task (performing arithmetic problems) occurred. Then, subjects proceeded into a cued recall task. During cued recall, subjects were presented with only the context items in a single random order at an 8 -sec rate and wrote their response to each contextual cue. The context items were the same that they had received at encoding. They were to recall the item that they had studied with that context item.

\section{RESULTS}

The mean number of words correctly recalled and the mean number of intrusions were subjected to analyses of variance (ANOVAs) with age, cuing condition, and presence or absence of integrating sentences as variables. The cued recall analysis yielded a main effect of age, $\mathrm{F}$ (1, $157)=75.46$, with mean recall of 18.17 for young and 10.68 for old. There was also a main effect of condition, $\mathrm{F}(2,157)=45.26$, with recall for control, interacting, and related conditions of 10.98, 12.10, and 20.19 respectively. In addition, a significant Age x Condition interaction was found, $F(2,157)=3.46$, as represented in Table 1 . Orthogonal decomposition of the interaction indicated that age differences were largest in the unrelated (control) condition compared to the other two. A further comparison indicated that the difference score for the interacting condition was not significantly different from the related condition $(p>.15)$. In addition to the orthogonal decomposition, a second set of contrasts was conducted. For both young and old adults, the unrelated control condition was compared to the interacting condition and to the related condition. For both groups, the related condition was significantly better than the control condition, whereas the interacting condition was significantly better than control for only the older adults, $\mathrm{t}(23)=1.83, \mathrm{p}<.05$. Thus, in general, the comparisons suggested that context was facilitative for both age groups. The reported effects, however, were more pronounced for the elderly in both the interacting and related conditions. The verbal integration manipulation was not involved in any significant main effects or interactions.

The analysis of intrusions indicated that the older adults made more intrusions than the young, $\mathrm{F}$ $(1,157)=15.56$, with means of 5.6 and 3.6 respectively. There was also a main effect of verbal integration, $\mathrm{F}(1,157)=4.93$, which occurred because subjects who listened to the verbal integrators made fewer intrusions than those who did not receive the sentences (means of 5.19 and 4.05). There was also a significant Age $x$ Integration interaction, $F(1,157)=4.96$, which occurred because young adults made more intrusions under control compared to integration conditions $\mathrm{t}(157)=1.82$ (means of 4.75 and 2.46), whereas old adults' means did not differ for 
the two conditions (5.60 and 5.60). Finally, there was a significant Age $\mathrm{x}$ Condition interaction, $\mathrm{F}(2,157)=3.38$ shown in Table 1 , which was significant because young adults' intrusion rates differed from older adults in the related condition, $\mathrm{t}(157)=3.10$, but not the other two. Care should be taken in interpreting any differences in intrusion rates, however, due to overall recall differences between the age groups. Because recall was lower in the old compared to the young, old subjects had more opportunities to intrude. In fact, when the number of items intruded is conditionalized upon the number of opportunities to intrude (number words studied - number recalled / number words studied), the proportion of intrusions for young and old does not differ, with mean conditional probabilities of .277 and .283 for young and old respectively. Thus, even though the absolute intrusion rate did differ as a function of age, when the correction for opportunity to intrude was applied, young and old did not differ.

\section{DISCUSSION}

The major findings from this experiment can be summarized as follows. First, there was evidence that age differences were largest when target and context were poorly integrated, and that well-integrated target-context relationships facilitated the recall of older adults more than young adults. Second, the old subjects benefited from both conceptual and perceptual integration, while the young subjects benefited only if there was a conceptual relationship between target and contextual cue. This pattern of findings suggests that the old adults, in fact, may have been more sensitive to context, not less sensitive, as suggested by the generalencoding point of view. Finally, there was no evidence that verbal integrators provided by the experimenter enhanced picture memory in either young or older adults.

The finding that age differences were most pronounced with unrelated cues confirms the integration hypothesis. That is, older adults were able to utilize well-integrated contextual information effectively, regardless of whether it was general (as in the semantic condition) or specific (as in the interacting condition). Perhaps of even more interest is the finding that integration improved memory for target pictures more for old than young. This is consonant with the effects reported by Canestrari (1968) in the verbal mode for paired-associate learning. Furthermore, because integration in this study was operationalized in two ways, both different from those used by Canestrari, it suggests that the integration effect is a reliable one that may generalize to different classes of stimuli and procedures. Thus, the present experiment provides evidence for convergent validity of the integration construct. The finding that integration in the cued recall task acted as a memory support is important, because no training or active strategy manipulation was required to achieve these effects in the older adults. Overall, these results suggest that perhaps more emphasis should be placed on understanding the role of passive contextual support in improving the memory of older adults, as well as determining whether there are simple training strategies which can be suggested to older adults to increase their sensitivity to the support provided by contextual manipulations, a position similar to that proposed by Craik (1988).

Craik (1988) proposed the environmental support hypothesis, which suggests that age differences are largest when little contextual support is provided for memory. Craik and McDowd (1987) provided support for this hypothesis when they found age effects to be larger for cued recall tasks which provide less contextual support compared to recognition tasks. The integration hypothesis is consonant with the environmental support hypothesis, and refines it further by 
specifying specific conditions under which context will be useful to elderly adults. That is, the mere provision of environmental support in the form of contextual cues will not facilitate memory in the elderly unless the cues are well- integrated with the target information, as suggested by our results.

One might argue that both the integration and the environmental support hypotheses are merely variants of the often- observed interaction of age with task difficulty. That is, age differences increase as tasks become more difficult (Salthouse, 1982). The importance of the integration hypothesis is that it specifies a priori relationships which will result in effective or ineffective use of context on the part of elderly adults, whereas the difficulty hypothesis results in a tautology. Difficult tasks are often defined post hoc by the magnitude of the observed age differences. Although one might still view the integration hypothesis as description rather than explanation (i.e., it is not clear what underlying structure or process accounts for the integration effect), it does increase the specificity and precision of predictions with respect to the interaction of age and contextual variables.

One aspect of the results that was somewhat surprising was the finding that the interacting condition did not facilitate young adults' memory. One possible interpretation of this finding is that young adults spontaneously provided visual or verbal integrations in the control condition so that no further facilitation accrued from the interacting context cues. It should also be noted that the facilitation effect appeared to be of a smaller magnitude for the elderly in the interacting condition compared to the related condition. Thus, although the two types of integration affected the elderly similarly, the interacting manipulation did not appear to be as powerful as the related manipulation. Perhaps further research on differences in the use of interacting compared to related integrators would yield systematic differences in their effectiveness for elderly adults.

The other finding in the present study that was unexpected was the weak effect of the verbal integrators during acquisition, particularly in light of Canestrari's (1968) findings, where such experimenter-provided integrators were found to be helpful. Canestrari did use longer presentation intervals which may have allowed subjects to utilize them more effectively; also, the primary target items were verbal rather than pictorial in the Canestrari study. However, Arenberg (1977) did use verbal descriptions of visual forms on the Benton Visual Retention test. He found, like Canestrari, that the verbal descriptors improved performance in older adults more than in the young. Like Canestrari, Arenberg used a long presentation interval. Thus, it would certainly be premature to conclude that verbal integrations are ineffective memory supports for pictorial information, as there are some precedents for this in the literature. Further research might be conducted to determine if verbal integrations might be more facilitative if they occurred after each stimulus had been presented rather than during presentation, or if integrations are better if they are self-generated (Treat \& Reese, 1976). Moreover, it would also be of interest to vary the format (picture or word) of both the targets and integrators within a single study.

Finally, the findings from this study suggest that a better understanding of how young and elderly adults use context to support memory may be obtained through the use of the integration construct. Based on the present findings, as well as others (Puglisi et al., 1988; Park et al., 1986, 1987), it is clear that elderly adults can use specific contextual information effectively to support memory. These findings are difficult to explain within the framework of the general encoding 
hypothesis. The general encoding hypothesis would predict the large age differences that were reported for unrelated items, but it would also predict that the interacting items would not facilitate memory in the elderly (due to the fact that they were semantically unrelated), in contrast to the reported findings. The interacting condition required the use of specific unrelated cues rather than general semantic information to facilitate memory. Both the general encoding and the integration hypothesis would predict that older adults could use semantic cues effectively. In sum, although the general encoding hypothesis would predict some of the results, it appears that the integration hypothesis is a better predictor of memory performance. Although the integration hypothesis may prove to have limitations upon further study, it does appear to be a promising approach to understanding the relationship among age, context, and memory.

\section{ACKNOWLEDGMENTS}

This research was supported by grant number ROI-AG-060625 from the National Institute on Aging, NIH.

The authors thank Katie Cherry for her assistance with data scoring and analysis. Address correspondence to Dr. Denise C. Park, Gerontology Center, University of Georgia, Athens, GA 30602.

\section{REFERENCES}

Arenberg, D. (1977). The effects of auditory augmentation on visual retention for young and old adults. Journal of Geronrology, 32, 19²-195.

Canestrari, R. E. (1968). Age changes in acquisition. In G. A. Talland (Ed.), Human aging and behavior. New York: Academic Press.

Craik, F. I. M. (1983). On the transfer of information from temporary to permanent memory. Philosophical Transactions of the Royal Society' London. Series B, 302, 3341-3359.

Craik, F. 1. M. (1988). Issues in attention, memory and aging. Paper presented at the Second Cognitive Aging Conference, Atlanta.

Craik, F. I. M., \& McDowd, J. (1987). Age differences in recall and recognition. Journal of Experimental Psychology: Learning' Memory' and Cognirion' J3, 474-479.

Craik, F. 1. M., \& Simon. E. (1980). The roles of attention and depth of processing. In L. W. Poon, J. L. Fozard, L. Cermak, D. Arenberg, \& L. W. Thompson (Eds.). New directions in memory and aging: Proceedings of the George Tolland memorial conference. Hillsdale. NJ: Erlbaum.

Duke University Center for the Study of Aging and Human Development. (1975). OARS Multidimensional Functional Assessment Questionnaire. Durham, NC: Author.

Fernandez, A.. \& Glenberg, A. (1985). Changing environmental context does not reliably affect memory. Memory \& Cognirion' 13' 333-345.

Gardner, E., \& Monge, R. (1977). Adult age differences in cognitive abilities and educational background. Experimenral Aging Research, 3. 337-383.

Hess, T. M. (1984). Effects of semantically related and unrelated contexts on recognition memory of different-aged adults. Journal of Gerontology, 39' 444-451.

Howard, D. V., McAndrews, M. P.. \& Lasaga, M. I. (1981). Semantic priming of lexical decisions in young and old adults. Journal of Gerontology, 36, 707-714.

Park, D. C., Puglisi, J. T.. \& Smith, A. D. (1986). Memory for pictures: Does an age-related decline exist? Journal of Psychology and Aging,1, 11-17. 
Park. D. C., Puglisi, J. T., Smith, A. D.. \& Dudley. W. N. (1987). Cue utilization and encoding specificity in picture recognition by older adults. Journal of Geronrology, 42, 423-425.

Puglisi. J. T., Park, D. C., Smith. A. D. \& Dudley. W. N. (1988). Age differences in encoding specificity. Journal of Geronrology, 43, 145151.

Salthouse, T. A. (1982). Adult cognition. New York: Springer-Verlag. Smith, S. (1979).

Remembering in and out of context. Journal of Experimental Psychology: Human Learning and Memory, 5, 460-471.

Snodgrass, J. C., \& Vanderwart. M. A. (1980). Standardized set of 260 pictures: Norms of name agreement, image agreement, familiarity, and visual complexity. Journal of Experimenral Psychology: Human Learning \& Memory, 6, 174-215.

Treat, N. J., \& Reese, H. W. (1976). Age, imagery and pacing in paired-associate learning. Developmental Psychology, 12, 119-124.

Zaretsky, H., \& Halberstam. J. (1968). Age differences in paired-associate learning. Journal of Geronrology, 23, 165-168. 